\title{
Selecting communication media for distributed communities
}

\author{
E. Heeren \& R. Lewis* \\ University of Twente/Telematics Research Centre and Lancaster University ${ }^{*}$
}

\begin{abstract}
Within the 'Virtual Mobility and Distributed Laboratories' project three naturalistic case studies of distributed research communities were conducted with a focus on the communication media used. The findings provide insight into relationships between the different media that the communities selected, and the different activities to which these media contributed. It is suggested that these findings are also relevant for distributed groups in which collaborative learning is the primary aim. A framework is presented for understanding and recommending selections of media for particular kinds of tasks, which is derived by integrating Mediarichness Theory and Activity Theory. This framework indicates how task/media fit may be achieved while taking into account the evolving character of activities in a distributed community. Some implications of the framework for collaborative distance learning are highlighted.
\end{abstract}

Keywords: Activity Theory; Collaborative distance learning; Communication media; Distributed research groups; Mediarichness Theory

\section{Introduction}

The availability of advanced electronic communication functionality has provided enormous support to some research communities which are geographically separated (see Sanderson, 1994, for an overview). Similarly, such technologies have allowed various advanced designs for collaborative distance learning (McConnell, 1994; Heeren, 1996). Distributed collaborative groups, existing for either working or learning purposes, or both, have to make decisions on the selection of appropriate media to support their communication and collaboration. This paper presents insights gained on this issue from studying media use in three existing distributed research communities. Mediaselection decisions made in these communities could be related to characteristics of the tasks/activities in which the communities were engaged. At the same

Accepted: 10 February 1997

Correspondence: Dr. E. Heeren, Telematics Research Centre, P.O. Box 589, 7500 AN Enschede, The Netherlands. Email: E.Heeren@trc.nl 
time it was observed that these selections needed to evolve according to temporal, dynamic shifts in the community which might be self-imposed or responsive to external demands. These relationships may be usefully modelled by integrating Media-richness Theory with Activity Theory. Activity Theory with its rich, holistic perspective on human activity provides context for concrete Media-richness Theory considerations. The result is a more comprehensive and applicable framework within which to explore further the design principles for distributed community support environments.

The first part of the paper introduces the 'Virtual Mobility and Distributed Laboratories' (VMDL) research project and the three naturalistic case studies conducted within this project. Then the relevant concepts of Activity Theory will be described, followed by those of Media-richness Theory, and thirdly an integration of these two theories will be suggested. Along the way, findings from the case studies will be used for illustration. The concluding section addresses the applicability of these theories to distributed learning groups.

\section{Studying media use in distributed research communities}

In Europe, distributed research communities have become of particular interest since the European Commission has stimulated collaborative pan-European research. Such international project groups are often inter-disciplinary and include partners from both university and business. The rational for and motivation of such communities have been studied by Hughes and Christie (1995). Pan-European mobility of researchers is a related topic of interest.

In this context the VMDL project was supported by the European Commission under the DGXII programme 'Human Capital and Mobility'. Researchers from different disciplines relevant to the area of CSCW and from four institutions distributed over Europe formed the VMDL project team. The team functioned as a distributed research community itself. It set out to explore conditions necessary to support distributed research communities, with a special focus on the use of communication media (Lewis \& Collis, 1995). The project approach could be characterised as naturalistic and exploratory. It partly took the form of action research, as the partners applied the research methodologies to their own functioning, and iteratively and reflexively piloted the outcomes of the research.

The research was centred around the question: What factors influence the success of a distributed research community?

- where success was defined idiosyncratically, from the perspective of the individual both personally and as a community member, as the success of the community related to individual and group motivations;

- where factors related primarily to communication media, and to interactions of communication media with other factors, especially organisational structure of the community, nature of the research task, and member characteristics;

- where the distributed research communities considered closely were those which met occasionally face-to-face, and between face-to-face sessions continued working and communicating via, mostly simple, electronic 
communication channels - which is how most EC-project teams and many other distributed research groups work.

Communication media were defined as communication channels comprising both the technical means of communication and the method or structure of communication. Following this definition, face-to-face meetings were considered as a medium as well. Communication media were explored with a focus on:

- 'within media' referring to the characteristics, methods and effectiveness of a particular communication medium;

- 'between media' focussing on the complementarity of various communication media used;

- 'over time' referring to the changes of the use and effectiveness of media over time, both 'within media' and 'between media'.

\section{The case studies}

Three distributed research teams were selected for study due to the wide variation in their tasks, composition, management structure and life-span:

- Case 1: the VMDL Community. The VMDL project team had about 15 members from three universities and an independent research lab in four different countries, and was structured as a network of junior and senior researchers plus a project leader, all working closely together. It had an open, learning-oriented task, an evolving work structure, and a life-span of 14 months.

- Case 2: the European Project Group. This was an EC Third Framework DG XIII education and training project team involving five partners and about 30 researchers. Researchers collaborated locally, and were, both individually and as subteams, engaged in on-going communication and negotiation within the whole group. The group had a pre-defined task, a work-package structure, a project leader and work-package leaders, and a tight time schedule of 17 months.

- Case 3: the Community of Physicists. A group of about 40 physicists from a number of research institutes in Europe and North-America were involved in a long-term collaboration (over 10 years) on preparing and conducting a series of experiments. Local teams with a leader each worked on subtasks; they communicated with other teams to coordinate efforts, exchange results, and discuss future plans as necessary.

Data from these communities were collected through in-depth interviews conducted face-to-face, and document analysis. An additional data source was the record of interactions in a computer conference which was the main communication medium in the VMDL Community. Finally, the VMDL team was actively engaged in on-going reflective self-analysis and piloting of intermediary findings and ideas, discussions about which also served as data. Based on the case studies, three 'cross-case' analyses were carried out under specific themes drawn from the factors identified by the VMDL project team in 
addressing its overall research question:

- management and organisation of the community;

- the nature and stages of the research task;

- individuals and their engagement in the community.

It is the second of these which is the subject of this paper.

\section{Observations on media selection}

A major insight from the case studies was the importance of being able to use multiple media (see also Linard, 1995, for a similar finding). For the communities studied, these media included e-mail, computer conferencing, file transfer, fax, telephone, audio conferencing, video conferencing, and face-to-face meetings.

The foci 'within-media', 'between-media', and 'over time' were helpful in revealing interesting patterns. All communities preferred to use - and did use - different communication media for doing different things. These preferences changed over time, for reasons that could be related to changes in the nature of the tasks that were carried out while using the media. Furthermore the communities appeared to change the structure or the use of a single medium over time - again related to the changing nature of their tasks. The data revealed certain characteristics of each medium and allowed a comparison of media to be made (Table 1 ).

Table 1. Summary of results from the VMDL community: relationships between usages of different media.

\begin{tabular}{ll}
\hline Related Media & Character of Relationship \\
\hline $\begin{array}{l}\text { Open computer conferencing } \\
\text { - formal face-to-face meeting }\end{array}$ & $\begin{array}{l}\text { Communication in the computer conference forms an } \\
\text { important basis for decision-making face-to-face. }\end{array}$ \\
& $\begin{array}{l}\text { Face-to-face meetings can be used to plan the further } \\
\text { work carried out through the computer conference, and } \\
\text { make decisions on new topic structures for the computer } \\
\text { conference; }\end{array}$ \\
& $\begin{array}{l}\text { Face-to-face meetings may be used to establish criteria } \\
\text { on which to make decisions in the computer conference. }\end{array}$ \\
\hline $\begin{array}{l}\text { Formal and ad-hoc face-to-face } \\
\text { meeting - computer }\end{array}$ & $\begin{array}{l}\text { Face-to-face meetings can be useful for efficiently } \\
\text { developing shared understandings that could be built } \\
\text { conferencing }\end{array}$ \\
$\begin{array}{l}\text { Fax - computer conferencing in the computer conferencing. } \\
\text { or e-mail }\end{array}$ & $\begin{array}{l}\text { Fax may be used to share graphics and tables that were } \\
\text { part of texts such as report drafts, but that were more } \\
\text { complex to distribute through conferencing or e-mail. }\end{array}$ \\
\hline
\end{tabular}

The changes in preferred task/media combinations over time observed in the case studies led to a consideration of two theories relevant to media selection in CSCW settings: Activity Theory and Media-richness Theory. Linked together, they not only appeared to function as an attractive explanatory framework for the case-study findings, but also as a guiding framework for making mediaselection decisions in distributed communities. 


\section{Activity Theory: levels of focus of human activity}

Activity Theory was developed within the Russian tradition of social-cultural theoretical approaches. Activity Theory is characterised by a combination of objective (in the sense of: motivation-driven), ecological, and socio-cultural perspectives on human activity (Kaptelinin, 1996). It thus stresses intentionality, development and history of human processes, resulting in the consideration of the broad context and meaningfulness of human behaviour.

Nardi (1996) summarised the central concepts of Activity Theory. Human activity is composed of subject, object, actions, and operations. A subject is an individual or group engaged in an activity. A subject holds an object (in the sense of 'objective'), which motivates activity. Actions are goal-directed processes which the subject undertakes in line with the object, and involve routinised and unconscious behaviours: operations.

Linard (1995) described Leontiev's (1978) and Von Cranach's (1982) three hierarchical levels of human processes, each of which are related to a type of object. In the context of VMDL, the levels were interpreted as follows.

- The intentional level is oriented toward motives: needs, desires, or values. It is the level of global orientation which gives meaning to human processes. An example from distributed research communities is the development of a shared understanding of research questions to meet a desire to gain new insights relative to a particular research topic.

- The functional level is oriented to specific, conscious goals in the context of motives. It is the level of focussed organisational, planning and problemsolving processes in order to achieve a final goal or intermediate goals. An example from distributed research communities is the definition of the research methodology.

- The operational level is oriented to the practical conditions of actions. It is the level of practical routines which are a prerequisite to the conscious, purposeful actions at the functional level. An example from distributed research communities is the carrying out of an investigation according to the selected methodology.

As the examples above show, the intentional level provides context for the functional level, which in turn serves as a focus for the operational level. The framework is flexible, however, in that the level of a particular activity depends on the subject; for example, developing an internal communication and management structure may be quite routine for one research group, while it is a challenging activity for another.

An important characteristic of the framework is its dynamic nature, in that human processes may move from one level to another as a result of 'frustrations' relative to their objects. 'Frustrations' or contradictions may be the result of external factors changing. For example - continuing the illustration above if a problem is met in the practical routine of organising the community's communication (operational level), strategies for adapting the communication structure may be needed. Therefore a new goal is formulated, and the focus of human action moves up the hierarchy to the functional level. Once the new routine is agreed on, the action may take on an operational character again. 
Another example, observed in Case Study 1 , concerns alterations in community or subgroup membership which lead to a need for renegotiation of shared understanding, for example, of the specific research question to be asked in the collaborative research.

Furthermore contradictions may be a consequence of results from various lower-, same- or higher-level processes. For example, in the European Project Group (Case Study 2) results from various local negotiations - from the perspective of the project group: at an operational level - appeared to be contradictory once their combination was considered. This led to specific goalsetting and negotiation within the whole group (functional level).

Contradictions may also result from the interconnectedness of webs of activities in real-life situations (Kuutti, 1991): each community member participates in multiple activity frameworks, including multiple communities, and developments in one activity framework may influence and lead to contradictions in others. The shifts in the focus level of human activity could, for example, follow the changes indicated in Fig. 1.

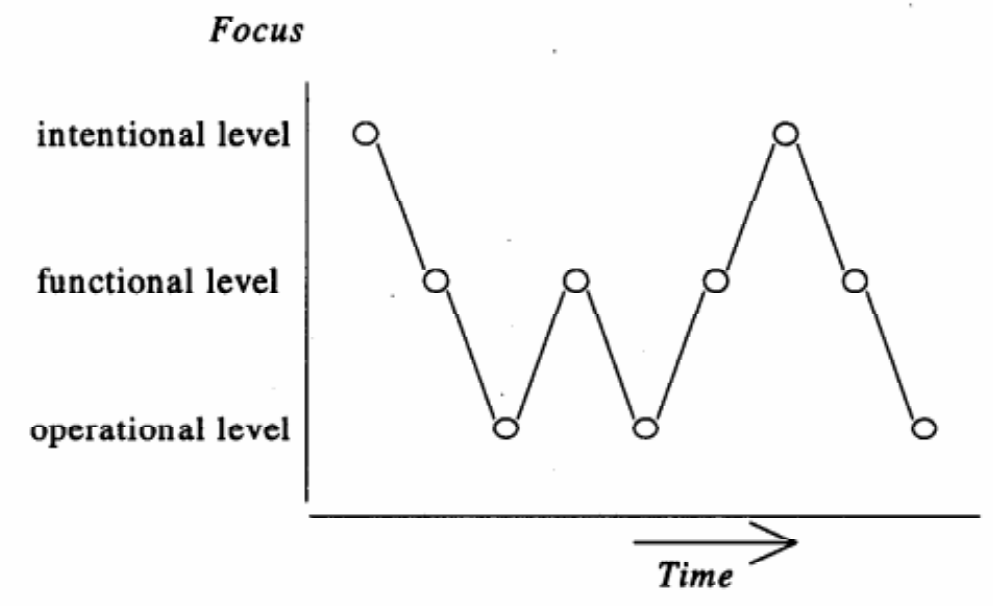

Fig. 1. Over time, the focus of human activity is at different levels.

Contradictions or 'frustrations' are thus a source for development on all three levels (Kuutti, 1991). In addition, this development provides human activity with intentionality and history which serves as a context for understanding human processes (Kaptelinin, 1996). This makes Activity Theory a rich and dynamic perspective on human activity which includes group work as occurring in distributed research and learning communities. This line of thought will be continued after considering a second conceptual framework: Media-richness Theory.

\section{Media-richness Theory: task/media fit}

Daft and Lengel (1984; see also Daft, Lengel \& Trevino, 1987), introduced an approach which considers information richness as a major factor in information processing and media selection by managers. They defined information richness 
as "the potential information-carrying capacity of data" (1984, p. 196), and stated that the medium used in communication deter-mines the potential richness of the information processed, and thereby the effect of a communication act.

According to this theory, media differ in their potential capacity for transmitting the meaning of information on four information-richness dimensions, so that they can be ranked from 'rich' to 'lean'. The dimensions are:

- Interactivity (speed of feedback). Rich media provide the opportunity for immediate feedback so that participants may adjust their messages "in response to signals of understanding or misunderstanding, questions, or interruptions" (Kraut et al., 1992, p. 378). Synchronous media are richer than asynchronous media in this respect.

- Multiple cues. Rich media allow a full range of verbal, paralinguistic, intonation, proxemic, and kinetic cues (Lim \& Benbasat, 1991) to convey not only the literal content of ideas, but also intensity and subtleties of meaning (Kraut et al., 1992). These 'surplus' cues are sometimes somewhat confusingly called 'social cues' (Daft et al., 1987; Farmer \& Hyatt, 1994). Lean media put constraints on the range of cues that may be used in communication. Face-to-face meetings are rich media in this respect, while text is lean.

- Language variety addresses the range of meaning that language symbols may convey (Farmer \& Hyatt, 1994). Numbers convey greater precision of meaning than natural language (Daft et al., 1987); and visual or graphic symbols carry a greater range of interpretations (Daft \& Lengel, 1984). Higher-variety languages are not only more ambiguous, but may also be used to organise a large amount of information (Farmer \& Hyatt, 1994) given that a shared understanding of the language has been established. Rich media such as video conferencing allow the use of a high variety language; lean media such as shared numeric databases restrict language use to low-variety language.

- Social-emotional cues, related to 'social presence'. Daft and colleagues (1987) describe rich media in this respect as media which permit communicators to have "personal feelings and emotions infuse the communication. Some messages may be tailored to the frame of reference, needs, and current situation of the receiver." (p. 358) For example, face-to-face meetings are richer than e-mail in this respect.

A medium's potential richness can be thought of as the sum of scores on each of these information-richness dimensions, though in particular settings a specific dimension may be more important than the others. In other words: 'medium richness' is defined by the information richness that a medium potentially transmits. 'Potentially' is added because the actual richness of a medium is determined by how users use it in a particular situation, which is influenced by, for example, their motivation or communication skills. 
Daft and Lengel (1984) further described how social systems have to process information for two purposes:

- to interpret the external environment;

- to coordinate internal activities.

These are tasks that may be characterised as involving both a particular degree of uncertainty (information is missing) and equivocality (information may be interpreted in different ways) (Daft et al., 1987). Two 'information tasks' are hence related to this (Daft \& Lengel, 1984):

- reduction of uncertainty: transmission of a sufficient amount of information;

- reduction of equivocality: developing shared understandings of external factors and frameworks of reference.

The complexity of group tasks in terms of group members' interdependence may be viewed as determined by the amount of uncertainty and equivocality involved: complex group tasks are those for which high levels of uncertainty and equivocality have to be reduced.

The relationship between potential richness of media used for group communication as determined by the four information-richness factors outlined above, and complexity of group tasks as a matter of uncertainty and equivocality, may be summarised in a task/media-fit hypothesis as shown in Fig. 2.

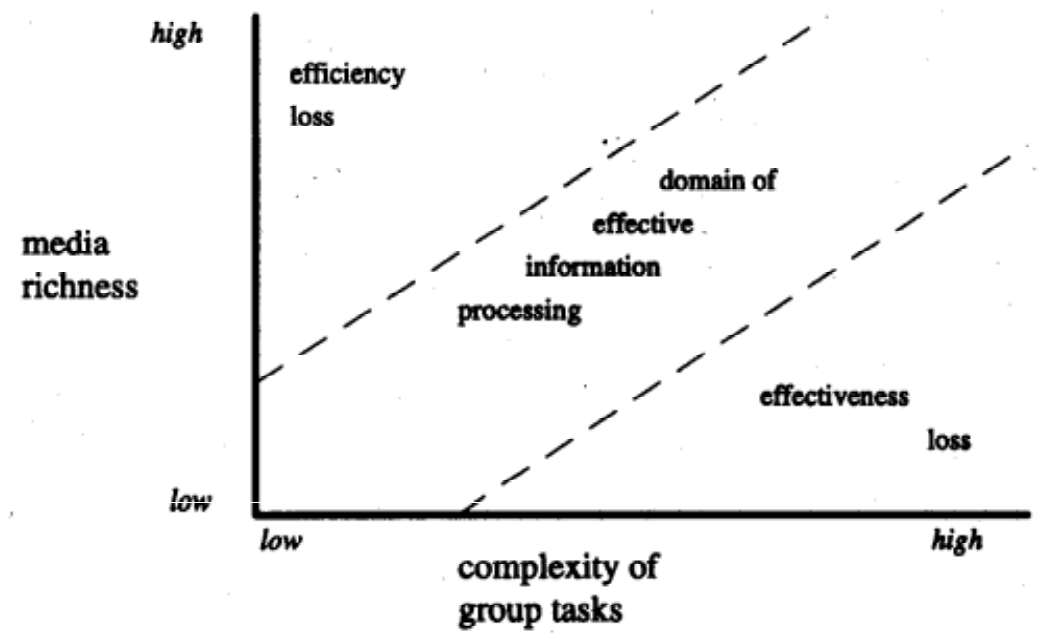

Fig. 2. Task/media-fit hypothesis (Heeren, 1996;

adapted from Daft \& Lengel, 1984; McGrath \& Hollingshead, 1993, 1994)

The amount of uncertainty reduction depends more on community members' willingness to share information, than on selected media of a certain richness. However, the media that are used by a community may influence the amount of equivocality that is potentially reduced in a community. As Fig. 2 shows, richer media allow the development of shared understanding if the need for equivocality reduction is high, while if this need is low, leaner media are both more effective and efficient. 
'Efficiency loss' in Fig. 2 refers to situations in which the media used provide a higher amount of information richness than necessary, resulting in 'noise'; information about relatively unambiguous matters may raise distracting questions of alternative interpretation. 'Effectiveness loss' on the other hand addresses the problem of too little information richness which makes it hard for participants to understand each other when different interpretations still exist.

The 'exact' positions of specific media on the vertical axis in Fig. 2 is unknown, but the principle of the task/media-fit hypothesis can be illustrated using examples from the case studies. For example, in all three communities studied, reaching common understandings with regard to the planning of the next stage of research (such as focussing on aspects of the research question), which may be considered a group task with a high need for equivocality reduction, was best done and was chosen to be done in a face-to-face setting, the richest medium available. When the VMDL community (Case Study 1) tried to develop research questions and a shared understanding of them through computer conferencing, it was found that equivocality could not be reduced to a sufficient degree (in fact, in the next face-to-face meeting it appeared that the shared understanding that all members thought they had, did not exist at all). Computer conferencing is a leaner medium than face-to-face meetings. It was hypothesized by the community members that the ineffectiveness of the computer conferencing in this respect was partly caused by the medium's textual nature (too few cues), and even more by the relatively low speed of feedback of the medium (too low interactivity) which, for example, did not enable 'speakers' to continuously or even quickly adapt to feedback supplied by 'listeners'.

\section{Integrating Media-richness Theory and Activity Theory}

In accordance with Activity Theory, three levels of processing relative to human activity may be distinguished. If human activity is limited to group tasks, and even further to the group members' interdependence in such tasks, a parallel may be drawn with a major concept used in Media-richness Theory: equivocality as determining group task complexity. This suggested relationship is presented in Fig. 3.

To illustrate Fig. 3, the development of a shared understanding of research questions is an example of a group activity at the intentional level, which is a highly equivocal and thus complex group task. The definition of a research methodology is an example of a group task at the functional level, and can be viewed to be of moderate equivocality. Conducting the investigation according to the selected methodology is a relatively unequivocal task at the operational level. 


\begin{tabular}{|cc|}
\hline Level of group activity & Equivocality in group tasks \\
\hline Intentional level & High equivocality \\
Functional level & \\
Operational level & Low equivocality \\
\hline
\end{tabular}

Fig. 3. Levels of human activity and equivocality of group tasks

Results from the case studies suggest evidence for the proposed relationships. For example, in the European Project Community addressed in Case Study 2, the community's work started off at the intentional level, with face-to-face workshops in which a shared understanding of the project's aims was developed. Activities at the functional level such as the planning of methodology and decision-making structure were also performed in such meetings. However, the leaner medium of computer conferencing appeared effective for operational activities carried out according to those plans, such as exchange of locally created products and negotiation of relationships between these according to 'rules' derived earlier at face-to-face meetings. However, when problems occurred in these operational activities which revealed that the strategy was not appropriate, the group's focus of activity shifted to the functional level again, and a richer medium was required: a telephone call in case of minor equivocality, or a face-to-face meeting in case of more complex problems - perhaps even differences in shared understanding at the intentional level.

Another example comes from Case Study 3 (the Community of Physicists). In this community face-to-face meetings were also held for activities at the intentional and functional levels, for which a high or moderate need for reduction of equivocality in understandings was experienced. Much of the work in this community was, from a group perspective, at an operational level. The results of the strategical planning carried out earlier gave context to operational activities; these could be handled locally, and exchange of the results of such activities could be shared by relatively leaner media such as email and document transfer without resulting in problems of different understandings across sites. However, when strategical issues needed to be renegotiated and the focus thus shifted to the functional level, a richer medium was required and often the telephone was used. Telephone allows verbal information to be trans-mitted, but not higher-variety languages; therefore, if for example graphics were required to reduce equivocality sufficiently, file exchange was chosen as an additional medium to achieve the required information richness. 


\section{Media selection in distributed research communities}

Integrating Media-richness Theory with Activity Theory gives the former a context, including a developmental character. The result is a dynamic framework for understanding and recommending task/media combin-ations. Such task/media combinations seem to be an important factor for the success of a distributed research community.

The framework suggests that relatively rich media should be chosen for mediating group activities at the intentional level in order to achieve effective equivocality reduction, while relatively lean media should be chosen for supporting group activities at the operational level to avoid efficiency losses. As group activities at all levels occur in the work of distributed research communities, this implies that such communities require multiple media for mediating their communication and collaboration in different phases of their work. Examples from the case studies show that using a rich medium such as face-to-face meetings is important in the startup phase of a project, so that shared understandings at the intentional level can be developed effectively. Results of higher-level activities will provide context for later lower-level activities which will thus involve less equivocality, and which will therefore require less rich media for effective and efficient collaboration.

The framework described in this section appears useful for making mediaselection decisions in distributed research communities. However, locating Media-richness Theory within Activity Theory with its holistic character implies the usefulness of perspectives that consider other factors as well. The effectiveness and efficiency of the endeavours of a distributed group depend heavily on social, managerial and motivational factors. Hughes and Christie (1995) examined a number of transnational research projects and identified motivational factors and management structures which influenced the perceived value of distributed research teams. The VMDL case studies identified the importance of group management structures and how they need to reflect the communication needs and demands of the group members. Human moderation and leadership of group interactions are critical concerns which must be understood and expressed explicitly in order to maintain group cohesion. Failure to be explicit can lead to feelings of exclusion from decision making and to lower levels of personal commitment to the collaborative goals.

Although such personal and managerial factors determine the success of a distributed community to a large extent, the bottom line of their influence is provided by the media selected for carrying out the activities in the community. The framework introduced in this paper may guide distributed research communities in selecting effective and efficient media. Finally, because of the comprehensive character of Activity Theory, the framework also provides starting points for exploring relationships between Media-richness Theory and motivational and social factors important in the design of electronic support of distributed research communities. 


\section{Learning in distributed communities}

The findings quoted above indicate that communications technology can provide support at certain phases of activity in the context of work. However, there is little evidence that this support assists learning communities except where those activities are structured so as to reflect working practices. Activity Theory is beginning to identify critical features of effective distributed working communities and it may be useful to consider these and ways in which these characteristics can be helpful in creating frameworks for distributed learning communities.

An activity emerges or is set-up with a number of intentions. In a community this requires establishing a 'common' language with all members coming to be committed to a shared, explicit motive. In other words, the actions cannot be set along strict lines; there must be space for interpretation, negotiation and the establishment of group ownership of the motive.

In a learning community, if this intentional stage of activity is attempted without adequate opportunities for face-to-face interaction (e.g. through videoconferencing - which might soon be available with better function-ality), it will be necessary for the tutor to monitor this stage closely and be aware that some group members might become (or feel - which is just as bad) disenfranchised. Furthermore, it is essential that opportunities be provided for further 'rich' interactions for the group in order to maintain, revise and develop the initial intentions.

VMDL research outcomes indicate that during the activity related to a group task, the leadership/organisational structure must change. There are times when a flat, 'democratic' structure is adequate for decision making but at other times this structure has to change - someone has to take the lead, to become the leader and assist the collaborative process. "... groups were likely to achieve a good group product if they proved able to develop a framework of structured interdependence together with individual auto-nomy. This we argue is facilitated by the task design, but relies upon the emergence of a high-status pupil-coordinator..." (Hoyles et al., 1994, p. 214).

Organisation and leadership issues cannot be ignored if distributed learning communities are to function effectively; they require discussion within the group during face-to-face meetings and require follow-up, as necessary, by the tutor.

“Openness and tolerance, and the will to reflect on others' meanings, are even more important in CMC than in face-to-face discussions. The lack of non-verbal communications and face-to-face contact were obvious in situations where everything went wrong."

(Løth \& Køhler, 1995, p. 42)

\section{Conclusions}

The availability or choice of communication channels seemed to be critical in the creation and maintenance of the distributed research communities studied. It is suggested that an awareness of the potential and the limitations of each 
class of channel is also necessary when planning to set-up distributed learning communities. Further, it is also necessary to make choices based on the purpose of the communications and the value that they may have for individual members of the community. It is clear that tutors play a key role, not only in being instrumental in making appropriate channels freely available but in monitoring how they are being used and in taking corrective action to sustain fragile learning communities.

\section{Acknowledgements}

The authors wish to acknowledge the added stimulus to their research which was provided by the Human Capital and Mobility Programme of the European Commission (Contract: ERBCHRX - CT940526 with DG XII) and the universities at Lancaster and Twente. Equally they acknowledge the contribution of other members of the VMDL team in Lancaster, Twente, Geneva, Coimbra and at Neurope Lab for their real and virtual interaction in aspects of the collaborative work. From TRC, Peter Hinssen's comments were helpful in the final moulding of this paper.

\section{References}

Daft, R.L. \& Lengel, R.H. (1984) Information richness: A new approach to managerial behavior and organization design. Research in Organizational Behavior, 6, 191-233.

Daft, R.L., Lengel, R.H. \& Trevino, L. (1987) Message equivocality, media selection, and manager performance: Implications for information systems. MIS Quarterly, 11, 355-366.

Farmer, S.M. \& Hyatt, C.W. (1994) Effects of task language demands and task complexity on computer-mediated work groups. Small Group Research, 25, 331-366.

Heeren, E. (1996). Technology support for collaborative distance learning. CTIT Ph.D.thesis series No. 96-08. University of Twente, Centre for Telematics and Information Technology, The Netherlands.

Hoyles, C., Healy, L. \& Pozzi, S. (1994) Groupwork with Computers: an overview of findings. Journal of Computer Assisted Learning, 10, 4, 202-215.

Hughes, K. \& Christie, I. (1995) UK E European science policy: The role of co-operative research networks. Policy Studies Institute, London.

Kaptelinin, V. (1996) Activity theory: Implications for human computer interaction. In Context and consciousness: Activity theory and human computer interaction (ed. B.A. Nardi) pp. 103-112. MIT Press, Cambridge, MA.

Kraut, R., Galegher, J., Fish, R. \& Chalfonte, B. (1992) Task requirements and media choice in collaborative writing. Human-Computer Interaction, 7, 375-407.

Kuutti, K. (1991) Activity theory and its applications to information systems research and development. In Information systems research: Contemporary approaches and emergent traditions (eds. H.-E. Nissen, H.K. Klein \& R. Hirschheim) pp. 529-549. North-Holland, Amsterdam.

Leontiev, A.N. (1978) Activity, consciousness, personality. Prentice Hall, Englewood Cliffs, NJ.

Lewis, R. \& Collis, B. (1995) Virtual mobility and distributed laboratories: Supporting collaborative research with knowledge technology. In Innovative adult learning with innovative technologies (eds. B. Collis \& G. Davies) pp. 163-173. Elsevier Science, Amsterdam. 
Lim, F.J. \& Benbasat, I. (1991) A communication-based framework for group interfaces in computer-supported collaboration. In Proceedings of the 24th Hawaiian Conference on System Sciences (pp. 610-620). IEEE, Los Alamitos, CA.

Linard, M. (1995) New debates on learning support. Journal of Computer Assisted Learning, 11, 4, 239-253.

Løth, S. \& Køhler, J. (1995) The conditions for challenging each other's understanding in CMC (in Danish). Humanistic Informatics, Open Learning, Aarlborg University.

McConnell, D. (1994) Implementing Computer Supported Cooperative Learning. Kogan Page, London,

McGrath, J.E. \& Hollingshead, A.B. (1993) Putting the "group" back in group support systems: Some theoretical issues about dynamic processes in groups with technological enhancements. In Group support systems: New perspectives (eds. L.M. Jessup \& J.S. Valacich) pp. 78-96. MacMillan, New York.

McGrath, J.E. \& Hollingshead, A.B. (1994) Groups interacting with technology: Ideas, evidence, and an agenda. Sage, Thousand Oaks, CA.

Nardi, B.A (1996) Studying context: A comparison of activity theory, situated action models, and distributed cognition. In Context and consciousness: Activity theory and human computer interaction (ed. B.A. Nardi) pp. 69-102. MIT Press, Cambridge, MA.

Sanderson, D. (1994) Mediated Collaborative Research? Claims, evidence and a case study of a distributed science group. Computer Supported Cooperative Work, 2, 1-2, 41-66.

Von Cranach, M. (1982) The psychological study of goal-directed action: Basic issues. In The analysis of action: Recent theoretical and empirical advances (eds. M. von Cranach \& R. Harre) pp. 35-73. Cambridge University Press, Cambridge.

\section{Forthcoming $J C A L$ Special Issue: \\ Computer-mediated Communications}

(Guest Editors: A. Tolmie \& S. Barbieri)

Inter-University cooperative learning: an exploratory study:

A. Calvani, P. Sorzio \& B.M. Varisco, Univerita di Firenze

Embedding resources for communication in hypertext course material:

Evaluating the Virtual Summer School:

C. Crook, University of Loughborough

$K$. Issroff $\mathcal{E} M$. Eisenstadt, University College, London $\mathcal{E}$ The Open University

Reaching for the Sky! P. Light $\mathcal{E}$ C. Colbourn, University of

Southampton

Investigating the dialectic between teaching, learning and technology:

E. McAteer $\mathcal{E}$ A. Tolmie, Universities of Glasgow and Strathclyde

Telematics and On-line Education:

G. Trentin, Instituto Tecnologie Didattiche, Genova

Monitoring participation in a CMC environment:

Gender, CMC and identity:

T. Wilson $\mathcal{E} D$. Whitelock, The Open University

S.J. Yates, The Open University 